IJMMS 27:2 (2001) 77-82

PII. S0161171201005907

http://ijmms.hindawi.com

(c) Hindawi Publishing Corp.

\title{
ON IDEALS OF IMPLICATIVE SEMIGROUPS
}

\author{
YOUNG BAE JUN and KYUNG HO KIM
}

(Received 2 October 2000)

\begin{abstract}
We introduce the notion of ideals in implicative semigroups, and then state the characterizations of the ideals.
\end{abstract}

2000 Mathematics Subject Classification. 20M12, 06F05, 06A06, 06A12.

1. Introduction. The notions of implicative semigroup and ordered filter were introduced by Chan and Shum [3]. The first is a generalization of implicative semilattice (see Nemitz [6] and Blyth [2]) and has a close relation with implication in mathematical logic and set theoretic difference (see Birkhoff [1] and Curry [4]). For the general development of implicative semilattice theory the ordered filters play an important role which is shown by Nemitz [6]. Motivated by this, Chan and Shum [3] established some elementary properties, and constructed quotient structure of implicative semigroups via ordered filters. Jun et al. [5] discussed ordered filters of implicative semigroups. In this paper, we introduce the notion of ideals in implicative semigroups. By introducing special subsets of an implicative semigroups, we provide a condition for the special subset to be an ideal. We establish two characterizations of ideals.

2. Preliminaries. We recall some definitions and results. By a negatively partially ordered semigroup (briefly, n.p.o. semigroup) we mean a set $S$ with a partial ordering $\leq$ and a binary operation $\cdot$ such that for all $x, y, z \in S$, we have

(1) $(x \cdot y) \cdot z=x \cdot(y \cdot z)$,

(2) $x \leq y$ implies $x \cdot z \leq y \cdot z$ and $z \cdot x \leq z \cdot y$,

(3) $x \cdot y \leq x$ and $x \cdot y \leq y$.

An n.p.o. semigroup $(S ; \leq, \cdot)$ is said to be implicative if there is an additional binary operation $*: S \times S \rightarrow S$ such that for any elements $x, y, z$ of $S$,

(4) $z \leq x * y$ if and only if $z \cdot x \leq y$.

The operation $*$ is called implication. From now on, an implicative n.p.o. semigroup is simply called an implicative semigroup.

An implicative semigroup $(S ; \leq, \cdot, *)$ is said to be commutative if it satisfies

(5) $x \cdot y=y \cdot x$ for all $x, y \in S$, that is, $(S, \cdot)$ is a commutative semigroup.

In any implicative semigroup $(S ; \leq, \cdot, *), x * x=y * y$ for every $x, y \in S$ and this element is the greatest element, written 1 , of $(S, \leq)$.

Proposition 2.1 (see [3, Theorem 1.4]). Let $S$ be an implicative semigroup. Then for every $x, y, z \in S$, the following hold:

(6) $x \leq 1, x * x=1, x=1 * x$,

(7) $x \leq y *(x \cdot y)$, 
(8) $x \leq x * x^{2}$,

(9) $x \leq y * x$,

(10) if $x \leq y$ then $x * z \geq y * z$ and $z * x \leq z * y$,

(11) $x \leq y$ if and only if $x * y=1$,

(12) $x *(y * z)=(x \cdot y) * z$,

(13) if $S$ is commutative then $x * y \leq(s \cdot x) *(s \cdot y)$ for all $s$ in $S$.

Now we note important elementary properties of a commutative implicative semigroup, which follows from (5), (6), and (12).

OBSERVATION 2.2. If $S$ is a commutative implicative semigroup, then for any $x, y$, $z \in S$,
(14) $x *(y * z)=y *(x * z)$,
(15) $y * z \leq(x * y) *(x * z)$,
(16) $x \leq(x * y) * y$.

3. Ideals of implicative semigroups. In what follows let $S$ denote an implicative semigroup unless otherwise specified. We begin by defining the notion of ideals of $S$.

DEFINITION 3.1. A subset $I$ of $S$ is called an ideal of $S$ if

(I1) $x \in S$ and $a \in I$ imply $x * a \in I$,

(I2) $x \in S$ and $a, b \in I$ imply $(a *(b * x)) * x \in I$.

EXAMPLE 3.2. Consider an implicative semigroup $S:=\{1, a, b, c, d, 0\}$ with Cayley tables (Tables 3.1 and 3.2) and Hasse diagram (Figure 3.1) as follows:

TABLE 3.1

\begin{tabular}{l|llllll}
\hline$\cdot$ & 1 & $a$ & $b$ & $c$ & $d$ & 0 \\
\hline 1 & 1 & $a$ & $b$ & $c$ & $d$ & 0 \\
$a$ & $a$ & $b$ & $b$ & $d$ & 0 & 0 \\
$b$ & $b$ & $b$ & $b$ & 0 & 0 & 0 \\
$c$ & $c$ & $d$ & 0 & $c$ & $d$ & 0 \\
$d$ & $d$ & 0 & 0 & $d$ & 0 & 0 \\
0 & 0 & 0 & 0 & 0 & 0 & 0 \\
\hline
\end{tabular}

TABLE 3.2

\begin{tabular}{l|llllll}
\hline$*$ & 1 & $a$ & $b$ & $c$ & $d$ & 0 \\
\hline 1 & 1 & $a$ & $b$ & $c$ & $d$ & 0 \\
$a$ & 1 & 1 & $a$ & $c$ & $c$ & $d$ \\
$b$ & 1 & 1 & 1 & $c$ & $c$ & $c$ \\
$c$ & 1 & $a$ & $b$ & 1 & $a$ & $b$ \\
$d$ & 1 & 1 & $a$ & 1 & 1 & $a$ \\
0 & 1 & 1 & 1 & 1 & 1 & 1 \\
\hline
\end{tabular}

We know that $\{1, a, b\}$ is an ideal of $S$, but $\{1, a\}$ is not an ideal of $S$, since ( $a *(a *$ $b)) * b=b \notin\{1, a\}$. 


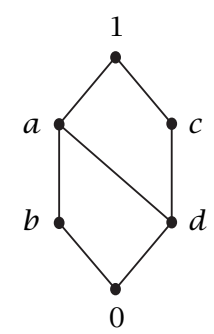

FIGURE 3.1

LEMMA 3.3. Every ideal of $S$ contains 1.

Proof. The proof follows from (6) and (I1).

LEMmA 3.4. If $I$ is an ideal of $S$, then $(a * x) * x \in I$ for all $a \in I$ and $x \in S$.

Proof. The proof follows by taking $b=a$ and $a=1$ in (I2).

COROLLARY 3.5. Let $I$ be an ideal of $S$. If $a \in I$ and $a \leq x$, then $x \in I$.

Proof. Let $a \in I$ and $x \in S$ be such that $a \leq x$. Using (6) and Lemma 3.4, we have $x=1 * x=(a * x) * x \in I$. This completes the proof.

LEMMA 3.6. Let I be a subset of $S$ such that

(I3) $1 \in I$,

(I4) $x *(y * z) \in I$ and $y \in I$ imply $x * z \in I$ for all $x, y, z \in S$. If $a \in I$ and $a \leq x$, then $x \in I$.

Proof. Let $a \in I$ and $x \in S$ be such that $a \leq x$. Then $x *(a * 1)=x * 1=1 \in I$ by (6) and (I3), and so $x=x * 1 \in I$ by (I4). This completes the proof.

The following is a characterization of ideals.

THEOREM 3.7. Let $S$ be a commutative implicative semigroup. A subset I of $S$ is an ideal of $S$ if and only if it satisfies conditions (I3) and (I4).

Proof. Let $I$ be an ideal of $S$. Then $1 \in I$ by Lemma 3.3. Let $x, y, z \in S$ be such that $x *(y * z) \in I$ and $y \in I$. Using Lemma 3.4, we get $(y * z) * z \in I$. It follows from (6), (15), and (I2) that

$$
x * z=1 *(x * z)=(((y * z) * z) *((x *(y * z)) *(x * z))) *(x * z) \in I .
$$

Conversely, assume that $I$ satisfies conditions (I3) and (I4). Let $x \in S$ and $a \in I$. Since $x *(a * a)=x * 1=1 \in I$ by (I3), it follows from (I4) that $x * a \in I$, that is, (I1) holds. Since $(a * x) *(a * x)=1 \in I$, we have $(a * x) * x \in I$ by (I4). Note from (15) that

$$
((a * x) * x) *((b *(a * x)) *(b * x))=1,
$$

that is,

$$
(a * x) * x \leq(b *(a * x)) *(b * x)
$$

for all $b \in I$. Thus, by Lemma 3.6, we have $(b *(a * x)) *(b * x) \in I$. Using (I4), we conclude that $(b *(a * x)) * x \in I$ which proves (I2). Hence $I$ is an ideal of $S$. 
For any $u, v \in S$, consider a set

$$
S(u, v)=\{z \in S \mid u *(v * z)=1\} .
$$

In Example 3.2, the set $S(1, a)=\{1, a\}$ is not an ideal of $S$. Hence we know that $S(u, v)$ may not be an ideal of $S$ in general.

THEOREM 3.8. Let $S$ satisfy the left self-distributive law under $*$, that is, $x *(y * z)=$ $(x * y) *(x * z)$ for all $x, y, z \in S$. For any $u, v \in S$, the set $S(u, v)$ is an ideal of $S$.

Proof. Let $x \in S$ and $a, b \in S(u, v)$. Then

$$
\begin{aligned}
u *(v *(x * a)) & =(u *(v * x)) *(u *(v * a))=(u *(v * x)) * 1=1, \\
u *(v *((a *(b * x)) * x)) & =(u *(v *(a *(b * x)))) *(u *(v * x)) \\
& =((u *(v * a)) *(u *(v *(b * x)))) *(u *(v * x)) \\
& =(1 *((u *(v * b)) *(u *(v * x)))) *(u *(v * x)) \\
& =(u *(v * x)) *(u *(v * x))=1 .
\end{aligned}
$$

Hence $x * a \in S(u, v)$ and $(a *(b * x)) * x \in S(u, v)$, which shows that $S(u, v)$ is an ideal of $S$.

LEMMA 3.9. Let $S$ be an implicative semigroup. If $y \in S$ satisfies $y * z=1$ for all $z \in S$, then $S(x, y)=S=S(y, x)$ for all $x \in S$.

Proof. The proof is straightforward.

EXAMPLE 3.10. Let $S:=\{1, a, b, c, d\}$ be an implicative semigroup with Cayley tables (Tables 3.3 and 3.4 ) and Hasse diagram (Figure 3.2 ) as follows:

TABLE 3.3

\begin{tabular}{l|lllll}
\hline$\cdot$ & 1 & $a$ & $b$ & $c$ & $d$ \\
\hline 1 & 1 & $a$ & $b$ & $c$ & $d$ \\
$a$ & $a$ & $a$ & $d$ & $c$ & $d$ \\
$b$ & $b$ & $d$ & $b$ & $d$ & $d$ \\
$c$ & $c$ & $c$ & $d$ & $c$ & $d$ \\
$d$ & $d$ & $d$ & $d$ & $d$ & $d$ \\
\hline
\end{tabular}

TABLE 3.4

\begin{tabular}{l|lllll}
\hline$*$ & 1 & $a$ & $b$ & $c$ & $d$ \\
\hline 1 & 1 & $a$ & $b$ & $c$ & $d$ \\
$a$ & 1 & 1 & $b$ & $c$ & $d$ \\
$b$ & 1 & $a$ & 1 & $c$ & $c$ \\
$c$ & 1 & 1 & $b$ & 1 & $b$ \\
$d$ & 1 & 1 & 1 & 1 & 1 \\
\hline
\end{tabular}




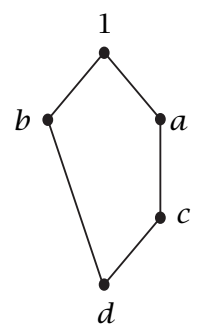

FIGURE 3.2

It is easy to check that $S$ satisfies the left self-distributive law under $*$, that is, $x *(y * z)=(x * y) *(x * z)$ for all $x, y, z \in S$. By Lemma 3.9 we have $S(x, d)=$ $S(d, x)=S$ for all $x \in S$. Furthermore we know that $S(1,1)=\{1\}, S(1, a)=S(a, 1)=$ $S(a, a)=S(a, b)=\{1, a\}, S(1, b)=S(b, 1)=S(b, b)=\{1, b\}, S(1, c)=S(a, c)=$ $S(c, 1)=S(c, a)=S(c, c)=\{1, a, c\}, S(b, a)=\{1, a, b\}$, and $S(c, b)=S$ are ideals of $S$.

Using the set $S(u, v)$, we describe a characterization of ideals.

THEOREM 3.11. Let $S$ be a commutative implicative semigroup and let I be a nonempty subset of $S$. Then $I$ is an ideal of $S$ if and only if $S(u, v) \subseteq I$ for all $u, v \in I$.

Proof. Assume that $I$ is an ideal of $S$ and let $u, v \in I$. If $z \in S(u, v)$, then $u *(v *$ $z)=1 \in I$ and so $z=1 * z=(u *(v * z)) * z \in I$ by (I2). Hence $S(u, v) \subseteq I$.

Conversely, suppose that $S(u, v) \subseteq I$ for all $u, v \in I$. Note that $1 \in S(u, v) \subseteq I$. Let $x, y, z \in S$ be such that $x *(y * z) \in I$ and $y \in I$. Since

$$
(x *(y * z)) *(y *(x * z))=(y *(x * z)) *(y *(x * z))=1,
$$

we have $x * z \in S(x *(y * z), y) \subseteq I$. Applying Theorem 3.7, we conclude that $I$ is an ideal of $S$.

THEOREM 3.12. Let $S$ be a commutative implicative semigroup. If I is an ideal of $S$, then

$$
I=\cup_{u, v \in I} S(u, v) .
$$

Proof. Let $I$ be an ideal of $S$ and let $x \in I$. Obviously, $x \in S(x, 1)$ and so

$$
I \subseteq \cup_{x \in I} S(x, 1) \subseteq \cup_{u, v \in I} S(u, v)
$$

Now let $y \in \cup_{u, v \in I} S(u, v)$. Then there exist $a, b \in I$ such that $y \in S(a, b)$. It follows from Theorem 3.11 that $y \in I$. Hence $\cup_{u, v \in I} S(u, v) \subseteq I$. This completes the proof.

COROLLARY 3.13. If I is an ideal of a commutative implicative semigroup $S$, then

$$
I=\cup_{w \in I} S(w, 1)
$$




\section{REFERENCES}

[1] G. Birkhoff, Lattice Theory, 3rd ed., American Mathematical Society Colloquium Publications, vol. 25, American Mathematical Society, Rhode Island, 1967. MR 37\#2638. Zbl 153.02501.

[2] T. S. Blyth, Pseudo-residuals in semigroups, J. London Math. Soc. 40 (1965), 441-454. MR 31\#1211. Zbl 136.26903.

[3] M. W. Chan and K. P. Shum, Homomorphisms of implicative semigroups, Semigroup Forum 46 (1993), no. 1, 7-15. MR 93g:20127. Zbl 776.06012.

[4] H. B. Curry, Foundations of Mathematical Logic, McGraw-Hill Book, New York, 1963. MR 26\#6036. Zbl 163.24209.

[5] Y. B. Jun, J. Meng, and X. L. Xin, On ordered filters of implicative semigroups, Semigroup Forum 54 (1997), no. 1, 75-82. MR 98a:06022. Zbl 862.06005.

[6] W. C. Nemitz, Implicative semi-lattices, Trans. Amer. Math. Soc. 117 (1965), 128-142. MR 31\#1212. Zbl 128.24804.

Young BAE Jun: DePARTMENT OF MATHEMATics EDUCATION, GyeONGSANG NATIONAL UNIVERSITY, JINJU 660-701, KOREA

E-mail address: ybjun@nongae.gsnu.ac.kr

KyUng Ho Kim: Department of MATHematics, ChungJu National University, ChungJu 380-702, KOREA

E-mail address: ghkim@gukwon.chungju.ac.kr 


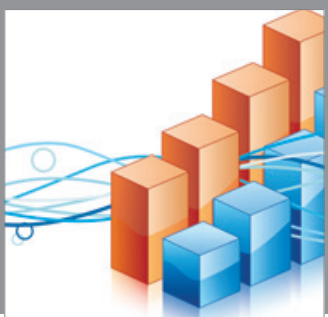

Advances in

Operations Research

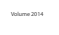

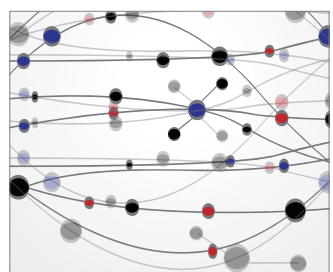

\section{The Scientific} World Journal
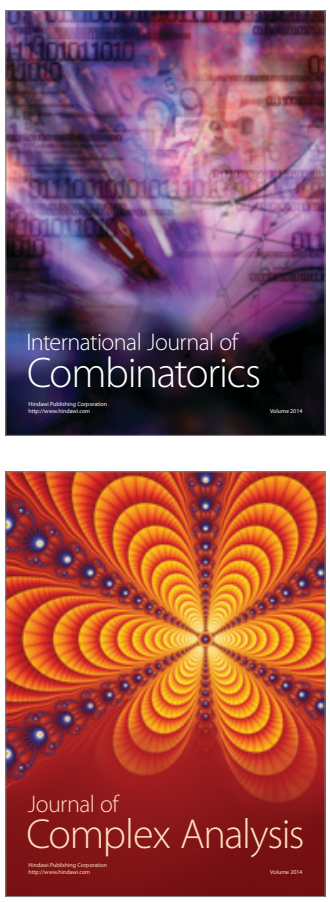

International Journal of

Mathematics and

Mathematical

Sciences
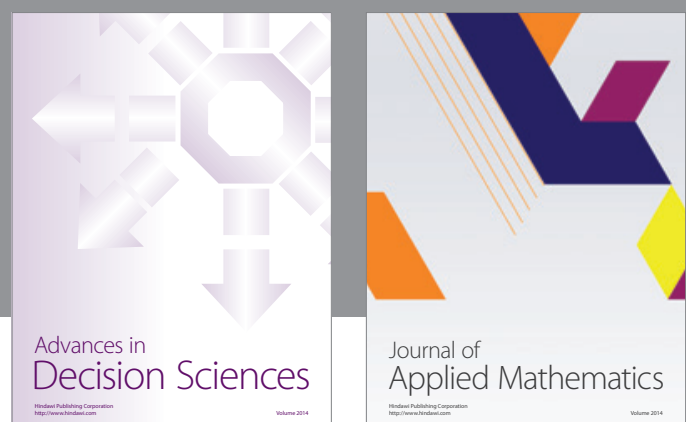

Journal of

Applied Mathematics
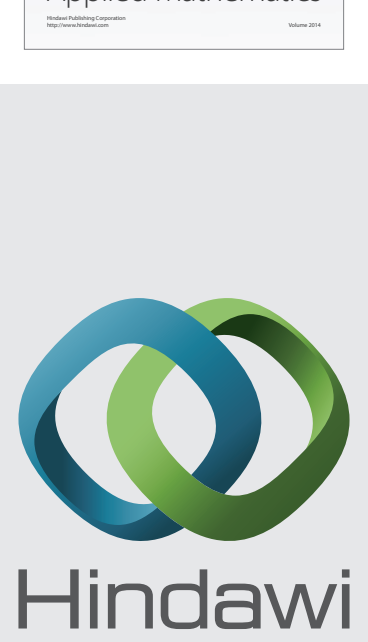

Submit your manuscripts at http://www.hindawi.com
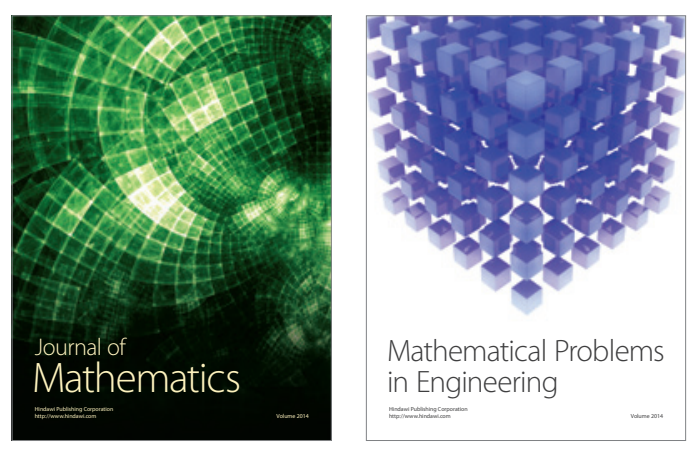

Mathematical Problems in Engineering
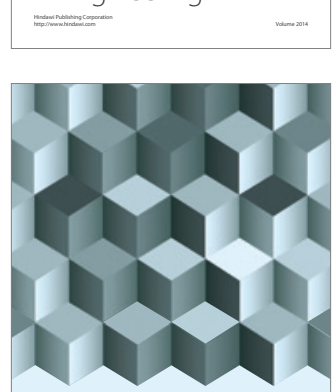

Journal of

Function Spaces
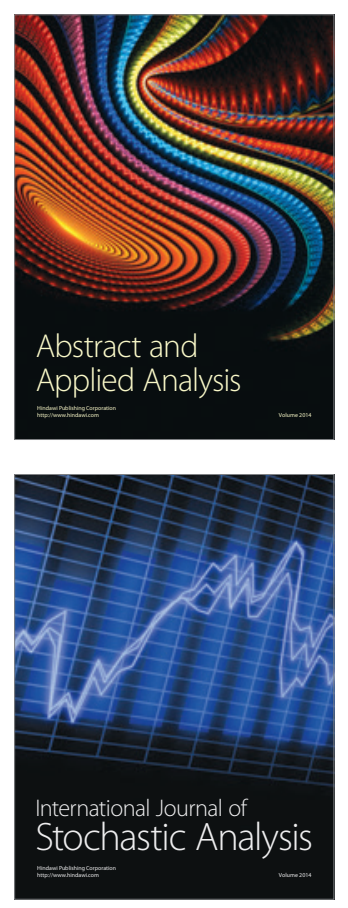

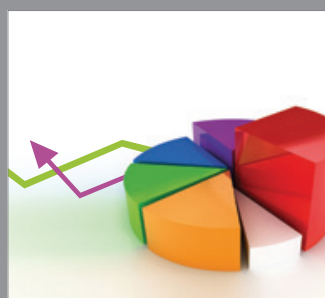

ournal of

Probability and Statistics

Promensencen
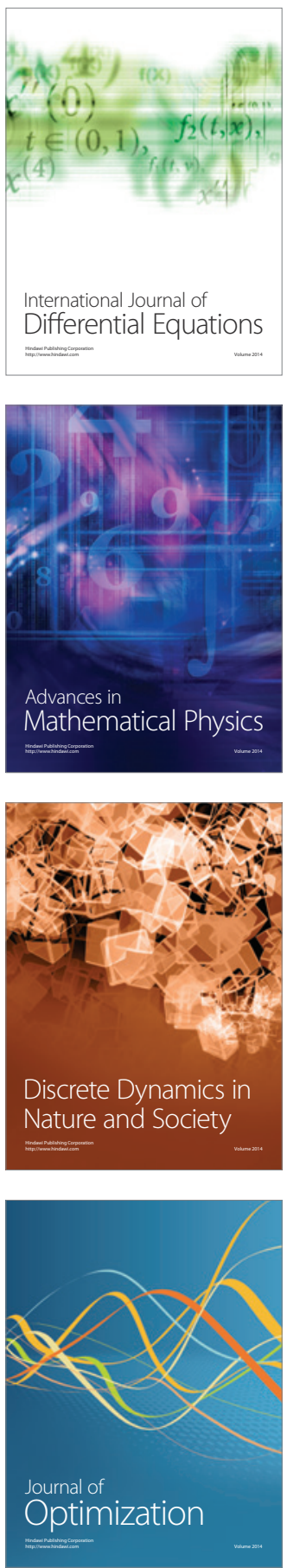